\title{
Resistance to Stresses and Reliability of Biological Systems: Insights for Genetic Studies of Human Aging, Health and Longevity
}

\author{
Anatoliy I. Yashin, Konstantin G. Arbeev, Liubov S. Arbeeva, Deqing Wu, Igor Akushevich, Alexander Kulminski, \\ Mikhail Kovtun, Ilya Zhbannikov, Svetlana V. Ukraintseva \\ Biodemography of Aging Research Unit, SSRI \\ Duke University \\ Durham, USA
}

\begin{abstract}
Connection between stress resistance and longevity in biological organisms is widely discussed and confirmed experimentally. Much less is known about the roles of genetic and non-genetic factors in regulation of such connection. Earlier studies emphasized that mechanism that realizes such connection involves interplay between processes of individual aging and external challenges. As a result of such interplay the parameters of the Gompertz mortality curve are negatively correlated. Such correlation has been also observed in the process of survival improvement in developed part of the world during the first part of the last century. The mortality decline was mainly due to favorable changes in external and living conditions as well as progress in health care. Surprisingly, similar pattern of survival changes is observed in the groups of individuals ranked with respect to the number of "longevity" alleles carried by individuals. We showed that this phenomenon can be interpreted as an increase in resistance to stresses and showed that similar effect is observed in reliability of technical systems when redundancy of their components increases. The availability of longitudinal data for genotyped individuals opens unique opportunity to address more sophisticated questions about roles of genetic and non-genetic factors in connection between aging, stress resistance and longevity in humans. For this purpose the dynamic model of human mortality and aging is used. We show how such model can be used in genetic analyses of fundamental processes of interaction between genetic and non-genetic factors to influence human longevity.
\end{abstract}

Key words-Stress resistance, aging, longevity, models for longitudinal data, stochastic process model of mortality and aging

\section{INTRODUCTION}

Connections between stress resistance and longevity in biological organisms are widely discussed and experimentally confirmed. The biological mechanisms involve responses to stress-signals that reached different levels of organism's biological hierarchy. This means that organism's response to stress results from integrating responses of physiological systems, organs, tissues, cells, as well as genetic factors. Although the entire mechanism of such hierarchical response remains elusive, some important connections were described and investigated. One such connection is outlined in Strehler and Mildvan [1] who showed that human mortality rate between ages 30 and 85 (approximated by the Gompertz's curve) can be represented as a result of interplay between aging related decline in resistance to stresses and the process of external challenges (stresses of life). Strehler and Mildvan model predicted negative correlation between parameters of the Gompertz's curve which was detected in the analyses of demographic mortality data. Such correlation took place in the process of survival changes during the first part of the last century when survival functions followed so called "rectangularization pattern" of survival improvement. Figure 1 shows age patterns of survival improvement in female population in Switzerland during 1876-2011. The changes in male survival in this country, as well as in other developed countries during this period of time follow similar patterns [2].

One can see from Figure 1 that survival improvement in the first half of the last century followed the "rectangularization" pattern. This pattern is characterized by an increase in survival in which mean life span is increasing, the variance is declining, and the maximum life span remains about the same. These changes can be explained by a decline in the average magnitude of external challenges (stresses of life) over time $[2,3]$ as well as by an increase in individual's resistance to stresses [4]. In the second half of the last century the rectangularization pattern of survival improvement was replaced by an almost parallel shift of the entire survival curve to the right. The new pattern is characterized by an increase in the mean and in the maximum life span, and by almost no changes in the variance of life span distribution. At the first glance, such changes in survival have little or nothing to do with genes, because it is quite unlikely that genetic background of the country residents changed substantially during this relatively short period of time. The more detailed analyses, however, indicate that genes play important roles in such changes by transforming external challenges into the organism's biological responses. 


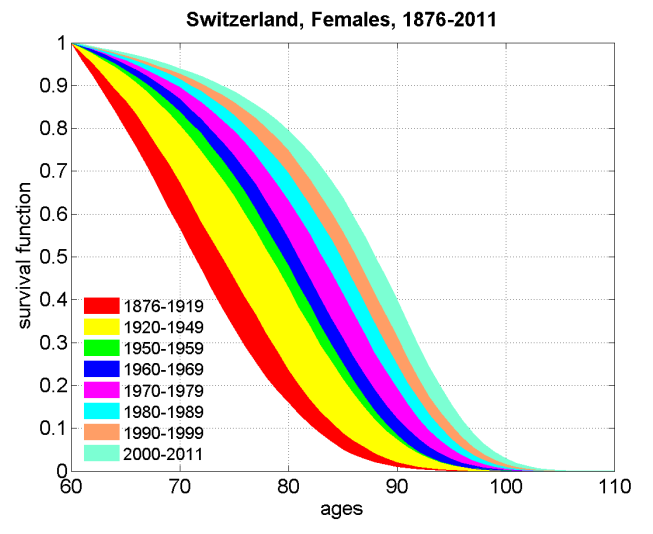

Fig. 1. Age patterns of survival improvement for females in Switzerland from 1876 till 2011. Survival functions are constructed using data from MPIDR/Berkeley Human Mortality Database (HMDB) for individuals survived age 60 years.

In the process of rectangularization of survival curve the mean lifespan was increasing, the lifespan variance was declining, and the maximum value remained about the same. In the second half of the $20^{\text {th }}$ century the rectangularization pattern of survival improvement was replaced by an almost parallel shift of the entire survival curve to the right. The new pattern was characterized by an increase in the mean and maximum lifespans, and by almost no changes in the variance of the lifespan distributions. The causes of such improvements were the subjects of intensive debates for several decades. Some researchers argued that social and economic developments were responsible for positive trends in health and survival [5-7]. The better quality of nutrition was emphasized in [8-10]. Others provided evidence of important roles of health care and advances in medicine [11-13]. Better understanding of the reasons for these trends can help us interpret the results of genetic analyses of human aging and longevity.

Reduction in stress load over time is likely to contribute to survival improvement. Stress response is the way how living organisms adjust their behavior and functioning to changing environments. There are evolutionarily developed connections between resistance to stresses and longevity [14-21]. Progress in health care and medicine may also have contributed to the continuing mortality decline. The development of health care and medicine enhanced each individual's limited capacities for coping with diseases and withstanding other deleterious consequences of human interactions with harmful and unfriendly environments. In other words, progress in health care and medicine enhanced each organism's resilience and resistance to stresses. Such contributions may have generated the rectangularization pattern of survival improvement seen in Fig. 1. Although the development of the health care industry followed basic economic principles, this system should also function in concordance with internal biological and physiological processes regulated by genes and modulated by external conditions. The genetic factors involved in the mediation of the effects of medical interventions on health status and lifespan are likely to include those dealing with common responses to stress, as well as intervention-specific genetic factors. Hence, the genetic polymorphisms that were able to efficiently interact with medical and health care interventions affecting variability in health and longevity are likely to be detectable in genetic association studies of those traits. Several recent publications provide evidence that a balanced diet rich in vitamins and other important nutrients contributes to an increase in individual resistance to stresses $[22,23]$ and, hence, in lifespan and survival. The metabolism of different nutrients may involve genes from different signaling and metabolic pathways [24]. Genetic variants that contribute to variability in health and lifespan in response to consumption of a given spectrum of nutrients are likely to be detected in GWAS of human lifespan and other health and longevity related traits. Also, improvements in social and economic status provide better access to health care and high quality nutrition, leading to additional improvements in lifespan and survival.

All external factors discussed above dynamically interact with the aging processes in individuals comprising each human population to produce the age patterns of mortality rates for the various birth cohorts. As mentioned in Section $\mathrm{C}$, a remarkable property of such interaction is that the resulting mortality rates between ages 30 and 85 always follow the Gompertz $\mu(x)=a e^{b x}$ or Gompertz-Makeham $\mu(x)=c+a e^{b x}$ laws. Time trends in survival improvement affect the parameters of these curves. These changes were not arbitrary but followed remarkable regularity: the parameter $c$ declined during all years. The changes in the parameters $a$ and $b$ during the first half of the $20^{\text {th }}$ century showed strong negative correlations in all developed countries with parameter $a$ declining, and parameter $b$ increasing [3, 25].

Surprisingly similar rectangularization pattern took place in the groups of individuals ranked with respect to the number of "longevity" alleles in their genomes. Using 39 longevity alleles selected from data on the original Framingham Heart Study (FHS) cohort [26] we constructed polygenic score index that counts the number of detected genetic variants carried by each subject. This index was used for evaluating the joint influence of subsets of genetic variants on survival. We showed that this index explains about $20 \%$ of life span variance and is able to predict life spans in individuals from the offspring FHS cohort using genetic variants detected from the data on the original FHS cohort. To evaluate effects of different genetic doses on survival we divided participants of the original FHS cohort into sub-cohorts of individuals having different numbers of longevity alleles in their genomes. Then we evaluated the joint influence of subsets of genetic variants on the age patterns of mortality and survival in these sub- 
cohorts. We separately fitted the Gompertz model to the mortality data at available age ranges in the sub-cohorts, constructed the corresponding survival functions. We used the Strehler-Mildvan mortality model [1] to guide our analysis of how the Gompertz parameters are affected by the genetic factors.

Fig. 2 shows age patterns of survival functions evaluated for sub-cohorts of genotyped members of the original FHS cohort survived age 60 years and carrying different numbers of "longevity alleles". The arrow shows how the age pattern of survival functions change when the numbers of carried longevity alleles increases. Since the environmental conditions were the same for each group, the SM model linked better survival with higher initial values of the vitality function, suggesting that exceptional survival was likely to have a genetic background. The more longevity alleles that were carried by study participants the higher was their initial stress resistance (the initial value of the vitality function).

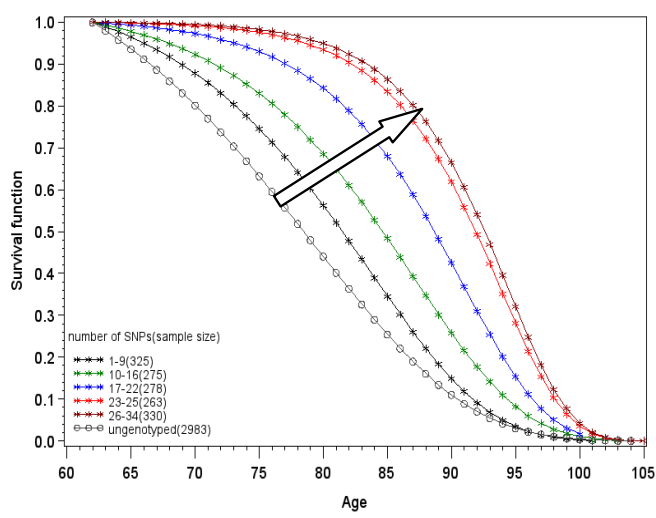

Fig.2. Age patterns of survival functions evaluated for sub-cohorts of genotyped members of the original FHS cohort survived age 60 years and carrying different numbers of "longevity alleles". The arrow shows how the age pattern of survival functions change when the numbers of carried longevity alleles increases.

In terms of genetic functions, the resistance to stresses is associated with DNA repair capacity, redundancy, and other functions that increase the resilience, or robustness, of each organism. A useful illustration of how increased resilience can influence survival derives from the model of saving lives [27]. An increase in the "number of lives being saved" for individuals in the population is interpreted as an increase in redundancy: for each life lost (premature death), the decedent has one or more "redundant" lives, depending on the relevant advances in medical technology [28].

The use of a measure of stress resistance (vitality) that is only hypothetical is a critical limitation of the SM model. To develop this concept for more realistic investigations, additional information and model extensions are required. The most promising sources of new information are the individuallevel longitudinal data files currently being assembled at multiple sites under NIH funding. Longitudinal data on aging, health, and longevity are needed to study the connections between mortality and resistance to stresses when such stresses are represented as deviations of physiological variables from their normal values.

Genetic data are needed for participants in longitudinal studies to analyze the roles of genetic factors in such connections. Both types of data are collected routinely in many longitudinal studies (e.g., FHS, LLFS, HRS, etc.). Comprehensive analyses of such data can be performed using the new integrative mortality models [29].

\section{STOCHASTIC PROCESS MODELS OF HUMAN MORTALITY AND AGING.}

Analyses of aging-related changes in physiological variables whose measurements are represented in longitudinal data require a model that represents the individual age trajectories for these variables. Such a representation is illustrated in Fig. 3. The scheme in Figure 3 is equivalent to the following stochastic differential equation with respect to the physiological variable $Y_{t}$ ( $t$ is age, $G$ denotes genotype/allele)

$$
d Y_{t}=a(t, G)\left(Y_{t}-f_{1}(t, G)\right) d t+B(t, G) d W_{t}
$$

This equation is solved using the initial condition $Y_{t_{0}}=Y_{0}$. The coefficients $a(t, G), f_{l}(t, G)$, and $B(t, G)$ characterize adaptive capacity, the effect of allostatic adaptation, and the strength of the stochastic component that modulates the effects of the Wiener process $\left(W_{\mathrm{t}}\right)$, respectively. Adaptive capacity is an important characteristic of aging because it tends to decline with age (homeostenosis) [30, 31]. The effect of allostatic adaptation integrates the influence of many external and internal challenges on physiological variable and it is used for evaluating allostatic load. Note that, although the notions of the effect of allostatic adaptation and allostatic load are conceptually close to those discussed [32] and [33], our mathematical description looks different from theirs because it is adapted to the structure of our model. 


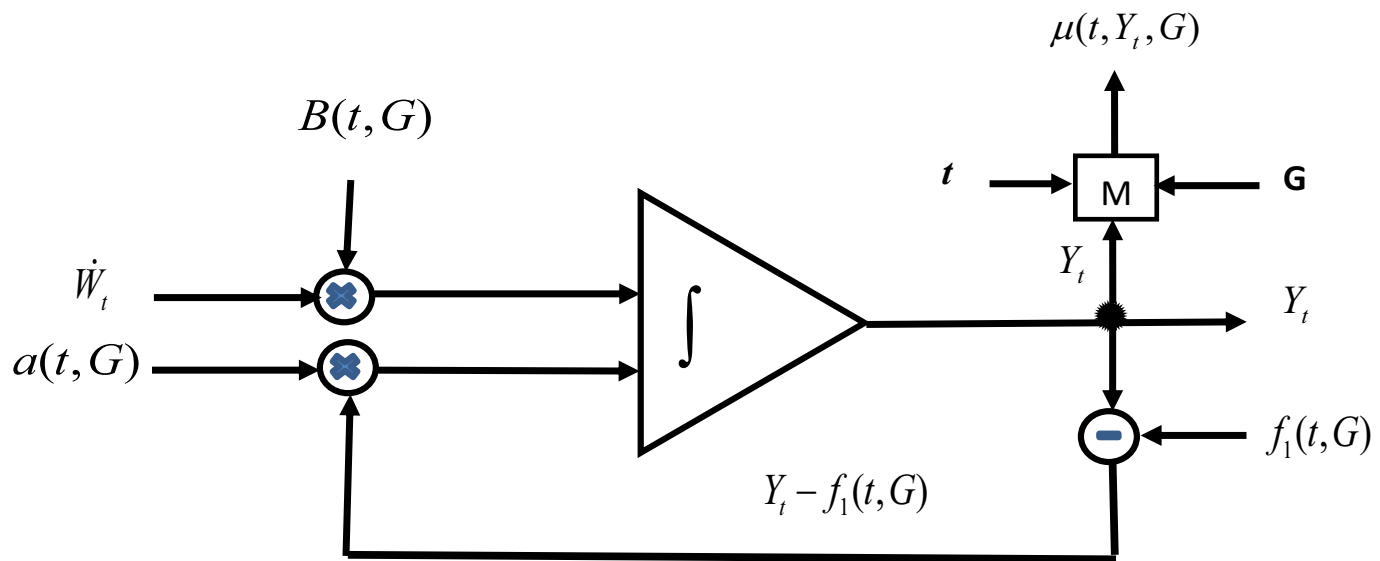

Fig. 3. Scheme illustrating the mechanisms that regulate age trajectories of individual physiological variables in the stochastic process model of human mortality and aging. The white noise $W_{t}$, enhanced by the coefficient $B(t, G)$ and the difference between the value of physiological variable $Y_{\mathrm{t}}$ and the effect of allostatic adaptation $f_{l}(t, G)$ multiplied by negative feedback coefficient $a(t, G)$ are added and integrated to produce the value of physiological variable $Y_{\mathrm{t}}$ at age $t$. This variable is used in a feedback regulation mechanism. Taken together with age, $t$, and genetic background, $G$, this variable is used for evaluating mortality risk at age $t$.

Allostatic load is defined as a difference between the effect of allostatic adaptation and the age-specific physiological norm. The need for conceptualizing the notion of norm has been widely discussed [34-36]. It is logical to define such a norm as the value of the physiological variable that minimizes the $U$-shaped mortality risk at a given age; in this case, the norm represents the optimal value of the variable. The use of the quadratic function for mortality risks allows us to capture the decline in stress resistance with age [37]. The age dynamics of influential factors (e.g., physiological variables) in connection with mortality risks have been described using a stochastic process model of human mortality and aging [38]. Recent extensions of this model incorporating hidden biomarkers of aging into the model's structure have been used in analyses of FHS longitudinal data on aging, health, and longevity; see [37] and the references therein.

\section{THE $U$-SHAPED MORTALITY RISK CAPTURES THE DECLINE IN STRESS RESISTANCE.}

Many epidemiological studies provide evidence of U- or Jshaped risks as functions of different physiological characteristics of health. Therefore, the use of such quadratic (U- or J-shaped) hazards in the analytic models is biologically meaningful [37]. The implications of using a quadratic form for the conditional hazard rate in analyses of aging and longevity from longitudinal data are illustrated in Fig. 4.

The model assumes that the normal values can be different at different ages. Individual physiological state can also change with increasing age. Figure 4 corresponds to the following representation of the hazard rate $\mu\left(t, Y_{t}, G\right)$ for individuals at age $t$ :

$$
\begin{aligned}
\mu\left(t, Y_{t}, G\right) & =\mu_{0}(t, G) \\
& +\left(Y_{t}-f_{0}(t, G)\right)^{*} Q(t, G)\left(Y_{t}-f_{0}(t, G)\right)
\end{aligned}
$$

Here $\mu_{0}(t, G)$ is a genotype-specific baseline hazard, $f_{0}(t, G)$ is the genotype-specific physiological norm, $Q(t, G)$ is a genotype-specific matrix regulating the width of the paraboloid (Fig. 4) at different times of life, and the symbol "*” denotes transposition. The advantage of using this approach for analysis of longitudinal data is that it allows all of the concepts and mechanisms of aging-related changes in physiological variables and other biomarkers mentioned above to be incorporated into the model under a common framework. The version of the stochastic process model (SPM) for analyses of genetic data was developed in [39]. This model and its extensions are described by a random Markov process that satisfies a diffusion type stochastic differential equation. This stochastic process is stopped at the random time associated with each individual's lifespan. The quadratic hazard assumption for the conditional mortality risk, given covariate values and certain regularity conditions, guarantees a Gaussian property for the conditional distribution of the covariate values at each given age. This property allows aging-related changes of the covariates to be described parsimoniously in terms of the first two conditional moments of a multidimensional Gaussian distribution. This distribution changes with increasing age. 


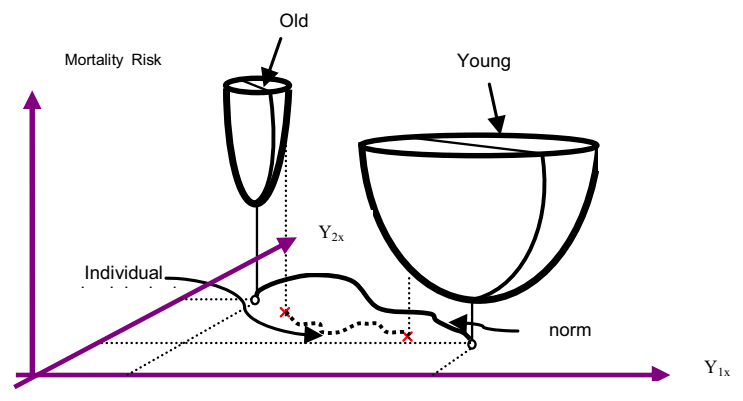

Fig.4. Illustration of hypothetical two-dimensional U-shaped mortality risks (quadratic hazards) considered as a function of two risk factors $\mathrm{Y}_{1 \mathrm{x}}$ and $\mathrm{Y}_{2}$ (e.g., physiological variables) for the 30 and 80 years old individuals. The width of each paraboloid characterizes resistance to stresses for the healthy young, healthy old and unhealthy young individuals. The coordinates of the minimal values of each paraboloid correspond to physiological norms.

\section{ESTIMATING GENETICS OF STRESS RESISTANCE FROM LONGITUDINAL DATA.}

One advantage of using longitudinal data in studies of genetic influences on longevity is the possibility of constructing empirical survival functions and age trajectories of physiological variables for the sub-cohorts of carriers and non-carriers of alleles or genotypes detected in association studies [40]. Such possibilities do not exist in case-control association studies when only two age groups of individuals are compared. The use of integrative mortality models opens an additional opportunity for studying how genetic factors associated with lifespan influence age trajectories of hidden biomarkers of aging, such as effects of allostatic adaptation, stress resistance, adaptive capacity, physiological norm, allostatic load, etc. These biomarkers are usually not measured in longitudinal studies. However, they modulate mortality risk and may influence age trajectories of other variables measured in the study. One such hidden biomarker characterizes resistance to stresses. In the integrative mortality models, this variable is represented by the coefficients describing the Ushaped mortality risk as a function of observed covariates. In the one-dimensional case, such mortality risk is represented by the following quadratic function

$$
\mu\left(t, Y_{t}, G\right)=\mu_{0}(t, G)+\mu_{1}(t, G)\left(Y_{t}-f_{0}(t, G)\right)^{2},
$$

where the notation $\mu_{1}(t, G)$ is used instead of $Q(t, G)$ in (2). The coefficient $\mu_{1}(t, G)$ characterizes stress resistance. It regulates the width of the U-shaped risk function (Fig. 4). When the value of this coefficient is small (the U-shape is wide) the risk function is less sensitive to small deviations of $Y_{t}$ from the norm. This is the case of better stress resistance.
When the value of this coefficient is high (the U-shape is narrow) the risk function is sensitive to small deviations of $Y_{t}$ from the norm. This is the case of worse stress resistance.

\section{DISCUSSION}

From the statistical point of view, human lifespan can be represented as a nonnegative random variable characterized by some probability distribution. Because influential factors may affect this distribution in thousands of different ways, many patterns of survival changes in response to changing environment are possible. Nonetheless, the changes in lifespan distribution during the last 2-3 centuries followed only two basic patterns. The first was associated with rectangularization of the survival curve (described by the SM correlation between the two Gompertz parameters $a$ and $b$ ). Then, in the middle of the $20^{\text {th }}$ century, this pattern was replaced by another pattern: an almost parallel shift of the entire survival curve to the right. This second pattern was characterized by an increase in the right tail of the lifespan distribution, which raised an important question: Did people with extreme longevity from distinct birth cohorts, i.e., who had lifespans from these right tails, have the same genetic backgrounds? One answer is "no" because lifespan is a multifactorial trait determined by genetic and nongenetic (environmental) factors, and human environments experienced substantial changes during the last century. At the same time, the fact that mechanisms of genetic regulation of human aging and longevity are evolutionary conserved indicates high possibility of detecting associations with genes from corresponding signaling and metabolic pathways that suppose function in different environments. Exposures to different types of environments may involve different genes and molecular biological pathways affecting lifespan regulation; hence, sets of genetic variants detected in GWAS of human longevity in independent populations may differ if people in these populations were exposed to different environments. Since exposures of populations to environmental conditions are hard to measure, one can expect difficulties in replication of findings using independent populations. If genetic factors involved in survival responses are sensitive to the spectrum of external challenges, one can expect that genetic variants contributing to exceptional longevity a hundred or more years ago may not be responsible for such traits today [41]. The concept of the "norm of reaction" can help us better understand what information is needed to clarify the effects of GxE interactions on lifespan.

\section{A. How do environmental changes influence aging, health, and longevity traits of individuals?}

It is clear that the changes in survival in Figure 1 resulted from the interplay among external conditions, genetic factors, ontogenetic, senescent, and other processes in individuals comprising the specific human populations. At first glance, it may appear that such changes have little or nothing to do with 
genes, because it is quite unlikely that the genetic backgrounds of the countries' residents changed substantially during the relatively short time periods involved. This point of view, however, is oversimplified because it assumes the existence of biological mechanisms through which the environment can modulate phenotypic traits-without involving genes. In reality, genes are always involved in such changes: they transform the signals from external challenges into the organism's biological and, ultimately, health/survival responses. The specific genes involved in forming these responses are likely to be detected in GWAS of human aging, health, and longevity. Thus, the genetic mechanisms involved in transforming environmental signals into metabolic and other biological responses account for the influences of external challenges on phenotypic traits.

The changes in survival in Fig. 1 demonstrate the effects of at least three types of gene/environment (GxE) interactions on lifespan. One type (e.g., better living and working conditions) reduced the frequency and average magnitude of environmental stresses. The second type enhanced stress resistance and resilience of human organisms using advances in medicine and health care. The third type improved individual stress resistance in response to better quality of nutrition and other components of metabolic processes. For all three cases, exposures to new conditions may have activated new genes and inactivated others. The genetic variants related to genes that interacted with different external conditions and contributed to changes in lifespan during an individual's life course are likely to be detected in GWAS of human aging and lifespan.

\section{B. The improvement of conceptual framework}

The improvement of conceptual framework for genetic analyses of human aging and longevity traits might substantially increase efficiency of genome wide association studies of these traits. Further development and implementation of the methods of systems biology in the analysis of data look promising [42]. Studying the effects of rare combinations of interacting common genetic variants (epistasis) or the absence of large numbers of harmful common variants that increase mortality risk at the old and the oldestold ages may make substantial contributions to our understanding of exceptional longevity. Exceptional longevity may also result from beneficial effects of interactions between common variants and environmental factors (GxE-interaction effects). The continuing improvement in survival (Figure 1) and unprecedented concurrent increases in the proportion of centenarians in developed countries provide strong evidence for age-specific GxE interaction effects on mortality risks. Many experts have offered (often controversial) opinions concerning the environmental factors responsible for these changes. Nonetheless, the details of the biological responses and mechanisms affecting the aging, health, and survival improvements still remain unknown.
The body of knowledge of various aspects of genetic influence on aging, health, and longevity continues to increase. These aspects include data on whole-genome sequencing, gene expression, epigenetics, etc. Such knowledge is gathered for individuals from longitudinal studies, case-control studies, patients in hospitals, volunteers, etc. Each new type of genetic data brings new information for studying how people age, how their health deteriorates, and how these changes affect lifespan. This information can be used to gain insight into alternative ways of slowing or postponing such health deterioration. Through analyses of these data, new roles of genetic factors in forming biological responses to external challenges will be elucidated. Questions regarding the dynamic interactions between external factors and biological mechanisms will be of crucial importance. Although dealing with such genetic data provides new opportunities for researchers, it does not automatically lead to understanding the connections between genetic factors and aging, health, and longevity traits; instead, the new opportunities create new challenges. The presence of many rare SNPs increases the number of statistical tests, which aggravates the problem of correcting for multiple testing. Dealing with low allelic frequencies requires larger sample sizes, which may be difficult for human studies. The need for developing effective strategies for maintaining good health for the elderly over longer time periods motivates the quest for deeper insights into the nature of individual aging processes and their relationships with health and survival outcomes. This requires information not only on individuals' genetic backgrounds but also on personalized aspects of genetic interactions with external forces and conditions, as well as on genetic and non-genetic influences on health and survival outcomes, and a better understanding of how those influences are mediated by physiological variables and other biomarkers. The data containing such information and the corresponding advanced methods needed for their analyses are being developed. The methods of systems biology combined with integrative statistical modeling and analyses of longitudinal data on genotyped individuals are likely to make substantial contributions to clarifying the connections among aging, health, and longevity [42].

\section{ACKNOWLEDGMENT}

Research reported in this publication was supported by the National Institute on Aging of the National Institutes of Health under Award Numbers R01AG046860, P01AG043352, and P30AG034424. The content is solely the responsibility of the authors and does not necessarily represent the official views of the National Institutes of Health. The Framingham Heart Study is conducted and supported by the National Heart, Lung, and Blood Institute (NHLBI) in collaboration with Boston University (Contract No. N01-HC-25195). This manuscript was not prepared in collaboration with investigators of the Framingham Heart Study and does not necessarily reflect the opinions or views of the Framingham Heart Study, Boston 
University, or NHLBI. Funding for SHARe Affymetrix genotyping was provided by NHLBI Contract N02-HL-64278. SHARe Illumina genotyping was provided under an agreement between Illumina and Boston University.

\section{REFERENCES}

[1] Strehler, B.L. and A.S. Mildvan, General theory of mortality and aging. Science, 1960. 132(3418): p. 14-21.

[2] Yashin, A.I., et al., The new trends in survival improvement require a revision of traditional gerontological concepts. Exp Gerontol, 2001. 37(1): p. 157-67

[3] Yashin, A.I., et al., New age patterns of survival improvement in Sweden: do they characterize changes in individual aging? Mech Ageing Dev, 2002. 123(6): p. 637-47.

[4] Yashin, A.I., et al., How genes influence life span: the biodemography of human survival. Rejuvenation Res, 2012. 15(4): p. 374-80.

[5] McKeown, T., R.G. Record, and R.D. Turner, An interpretation of the decline of mortality in England and Wales during the twentieth century. Popul Stud (Camb), 1975. 29(3): p. 391-422.

[6] Colgrove, J., The McKeown thesis: a historical controversy and its enduring influence. Am J Public Health, 2002. 92(5): p. 725-9.

[7] Link, B.G. and J.C. Phelan, McKeown and the idea that social conditions are fundamental causes of disease. Am J Public Health, 2002. 92(5): p. 730-2.

[8] Alfin-Slater, R.B., Nutrition and aging: introduction. Fed Proc, 1979. 38(6): p. 1993

[9] Kesteloot, H., Nutrition and life expectancy of populations. Acta Cardiol, 1993. 48(5): p. 441-2.

[10] Niculescu, M.D. and D.S. Lupu, Nutritional influence on epigenetics and effects on longevity. Curr Opin Clin Nutr Metab Care, 2011. 14(1): p. $35-40$.

[11] Mackenbach, J.P., The contribution of medical care to mortality decline: McKeown revisited. J Clin Epidemiol, 1996. 49(11): p. 1207-13.

[12] Abelson, P.H., Improvements in health care. Science, 1993. 260(5104): p. 11.

[13] Bunker, J.P., The role of medical care in contributing to health improvements within societies. Int J Epidemiol, 2001. 30(6): p. 1260-3.

[14] Kirkwood, T.L., P. Kapahi, and D.P. Shanley, Evolution, stress, and longevity. J Anat, 2000. 197 Pt 4: p. 587-90.

[15] Vermeulen, C.J. and V. Loeschcke, Longevity and the stress response in Drosophila. Exp Gerontol, 2007. 42(3): p. 153-9.

[16] Le Bourg, E., Hormesis, aging and longevity. Biochim Biophys Acta, 2009. 1790(10): p. 1030-9.

[17] Parsons, P.A., The ecological stress theory of aging and hormesis: an energetic evolutionary model. Biogerontology, 2007. 8(3): p. 233-42.

[18] Parsons, P.A., The limit to human longevity: an approach through a stress theory of ageing. Mech Ageing Dev, 1996. 87(3): p. 211-8.

[19] Calabrese, V., et al., Hormesis, cellular stress response and vitagenes as critical determinants in aging and longevity. Mol Aspects Med, 2011. 32(4-6): p. 279-304.

[20] Jazwinski, S.M., The retrograde response links metabolism with stress responses, chromatin-dependent gene activation, and genome stability in yeast aging. Gene, 2005. 354: p. 22-7.

[21] Epel, E.S. and G.J. Lithgow, Stress biology and aging mechanisms: toward understanding the deep connection between adaptation to stress and longevity. J Gerontol A Biol Sci Med Sci, 2014. 69 Suppl 1: p. S106.

[22] Sisodia, S. and B.N. Singh, Experimental evidence for nutrition regulated stress resistance in Drosophila ananassae. PLoS One, 2012. 7(10): p. e46131.

[23] Bosma-den Boer, M.M., M.L. van Wetten, and L. Pruimboom, Chronic inflammatory diseases are stimulated by current lifestyle: how diet, stress levels and medication prevent our body from recovering. Nutr Metab (Lond), 2012. 9(1): p. 32 .

[24] Barzilai, N., et al., The critical role of metabolic pathways in aging. Diabetes, 2012. 61(6): p. 1315-22.
[25] Yashin, A.I., et al., The new trends in survival improvement require a revision of traditional gerontological concepts. Experimental Gerontology, 2001. 37(1): p. 157-167.

[26] Yashin, A.I., et al., Joint influence of small-effect genetic variants on human longevity. Aging, 2010. 2(9): p. 612-620.

[27] Vaupel, J.W. and A.I. Yashin, Repeated resuscitation: how lifesaving alters life tables. Demography, 1987. 24(1): p. 123-135

[28] Yashin, A.I., et al., How genes influence life span: the biodemography of human survival. Rejuvenation Research, 2012. 15(4): p. 374-380.

[29] Yashin, A.I., et al., Joint analysis of health histories, physiological states, and survival. Mathematical Population Studies, 2011. 18(4): p. 207-233.

[30] Troncale, J.A., The aging process: Physiologic changes and pharmacologic implications. Postgraduate Medicine, 1996. 99(5): p. 111 $114,120-122$

[31] Lund, J., et al., Transcriptional profile of aging in C-elegans. Current Biology, 2002. 12(18): p. 1566-1573.

[32] Seeman, T.E., et al., Allostatic load as a marker of cumulative biological risk: MacArthur studies of successful aging. Proceedings of the National Academy of Sciences of the United States of America, 2001. 98(8): p. $4770-4775$.

[33] Karlamangla, A.S., B.H. Singer, and T.E. Seeman, Reduction in allostatic load in older adults is associated with lower all-cause mortality risk: MacArthur studies of successful aging. Psychosomatic Medicine, 2006. 68(3): p. 500-507.

[34] Palatini, P., Need for a revision of the normal limits of resting heart rate. Hypertension, 1999. 33(2): p. 622-625.

[35] Lewington, S., et al., Age-specific relevance of usual blood pressure to vascular mortality: a meta-analysis of individual data for one million adults in 61 prospective studies. Lancet, 2002. 360(9349): p. 1903-1913.

[36] Westin, S. and I. Heath, Thresholds for normal blood pressure and serum cholesterol. British Medical Journal, 2005. 330(7506): p. 1461-1462.

[37] Yashin, A.I., et al., The quadratic hazard model for analyzing longitudinal data on aging, health, and the life span. Physics of Life Reviews, 2012. 9(2): p. 177-188.

[38] Woodbury, M.A. and K.G. Manton, A random-walk model of human mortality and aging. Theoretical Population Biology, 1977. 11(1): p. 37-

[39] Arbeev, K.G., et al., Genetic model for longitudinal studies of aging, health, and longevity and its potential application to incomplete data. Journal of Theoretical Biology, 2009. 258(1): p. 103-111.

[40] Yashin, A.I., et al., How lifespan associated genes modulate aging changes: lessons from analysis of longitudinal data. Front Genet, 2013. 4: p. 3.

[41] Yashin, A.I., et al., Have the oldest old adults ever been frail in the past? A hypothesis that explains modern trends in survival. J Gerontol A Biol Sci Med Sci, 2001. 56(10): p. B432-42.

[42] Yashin, A.I. and S.M. Jazwinski, Aging and Health-A Systems Biology Perspective. Cytogenet Genome Res, 2014. 144(2): p. 77-154. 